\title{
An Improved Image Restoration Model Based on General Total Variation
}

\author{
Xue Gang FU \& Yong Jun XU \\ Sichuan University of Science \& Engineering Dept. of Electronic Engineering, Zigong, Sichuan
}

\begin{abstract}
The diffusion function of Aubert TV model does not satisfy the second-best condition, we improve the diffusion function to get better image restoration results. Experiments results show that the new model overcome the staircase effect in smooth area and preserving significant image details in the high frequency area. This model gain higher peak signal noise ratio and has good performance of image restoration. KEYWORD: Total variation; Diffusion function; Image gradient; Image restoration
\end{abstract}

\section{INTRODUCTION}

In all kinds of image acquisition system, the image transmission and transformation will cause the quality of image to derogate, such as blurring, distortion and noise disturbance, etc.. The images of high quality are need in most application of image, therefore, improving image quality and restoring image have very important significance. Since the 1990s, the partial differential equation image processing methods have obtained great development, and gradually become a very attractive topic. Perona and Malik's article [1] about partial differential equation is one of the most influent papers in the field of image processing, and attracts many researchers to study of partial differential equation image processing, after that, there are many improvements based on P-M diffusion model [2]-[3]. Under the same theoretical framework, Osher Rudin represents impulse filtering [4]-[5], and Rudin-Osher-Fatemi represents total variation image processing [6]-[7], all these researches show the necessity and the importance of differential equation in the applications of image processing.

Rudin put forward the Totally variational (Total Variation, TV) model of recovery method, which is more reasonable image restoration model to keep the image edge feature, this method is essentially based on the anisotropic diffusion, and it only spread along the orthogonal direction of the gradient image, so it preserve the image edge details at the same time of image restoring. But the noise of the smooth region in image is considered as edges in error that results in a false edge and ladder effect. Aubert puts forward a more general TV image restoration model [8] based on the diffusion function ${ }^{\circ}$. The model uses the different spread strength in different gradient regions, which can overcome the false edge, and preserve image details at the same time, howeyer, Aubert does not study of diffusion function $\phi(\cdot)$ in detail.

Adopting different diffusion function will get a different image restoration performance. In this article, based on analysis of the spread characters of Aubert's more general TV, the diffusion function is improved. Through the analysis of compare experiments, it is proved that the peak signal-to-noise ratio increases to a certain extent with the improved image restoration model in our article, and it has better performance.

\section{THE TV IMAGE RESTORATION MODEL}

Make $\mathrm{u}$ as the clear original images, ${ }^{u_{0}}$ as the blured image. Rudin Osher - Fatemi TV restoration model is described as follows:

$$
\min _{\boldsymbol{u}} J(\boldsymbol{u})=\int_{\Omega}|\nabla \boldsymbol{u}| d x d y+(\lambda / 2) \int_{\Omega}\left|\boldsymbol{u}-\boldsymbol{u}_{0}\right|^{2} d x d y
$$

Item 1 is the regularization item, and item 2 is the data fidelity term, which control the diffusion behavior $\lambda$ is reliability parameter. TV restoration model of the euler - Lagrange equation is:

$$
-\nabla \cdot\left(\frac{\nabla \boldsymbol{u}}{|\nabla \boldsymbol{u}|}\right)+\lambda\left(\boldsymbol{u}-\boldsymbol{u}_{0}\right)=0
$$

The corresponding diffusion equation can be written as: 


$$
\frac{\partial \boldsymbol{u}}{\partial t}=\nabla \cdot\left(\frac{\nabla \boldsymbol{u}}{|\nabla \boldsymbol{u}|}\right)-\lambda\left(\boldsymbol{u}-\boldsymbol{u}^{0}\right)
$$

It shows that TV diffusion model spreads only along the orthogonal direction of the image gradient diffusion [9], and the diffusion strength coefficient is $1 /|\nabla \boldsymbol{u}|$ So Aubert and Vese proposed various members of the anisotropic more general TV image restoration model:

$$
\min _{\boldsymbol{u}} J(\boldsymbol{u})=\int_{\Omega} \phi(|\nabla \boldsymbol{u}|) d x d y+(\lambda / 2) \int_{\Omega}\left|\boldsymbol{u}-\boldsymbol{u}_{0}\right|^{2} d x d y
$$

Where,

$$
\phi(|\nabla \boldsymbol{u}|)=\sqrt{1+|\nabla \boldsymbol{u}|^{2}}-1
$$

TV Image restoration model of the euler Lagrange equation is:

$$
-\nabla \cdot\left(\phi^{\prime}(|\nabla \boldsymbol{u}|) \frac{\nabla \boldsymbol{u}}{|\nabla \boldsymbol{u}|}\right)+\lambda\left(\boldsymbol{u}-\boldsymbol{u}^{0}\right)=0
$$

The corresponding diffusion equation can be written as:

$$
\frac{\partial \boldsymbol{u}}{\partial t}=\nabla \cdot\left(\phi^{\prime}(|\nabla \boldsymbol{u}|) \frac{\nabla \boldsymbol{u}}{|\nabla \boldsymbol{u}|}\right)-\lambda\left(\boldsymbol{u}-\boldsymbol{u}^{0}\right)
$$

Both sides of the euler - Lagrange equation multiply $\boldsymbol{u}-\boldsymbol{u}^{0}$, and integral in the entire image region $\Omega$, then obtain regularization parameters:

$$
\lambda=\frac{1}{\sigma^{2} \Omega} \int_{\Omega} \nabla \cdot\left[\phi^{\prime}(|\nabla \boldsymbol{u}|) \frac{\nabla \boldsymbol{u}}{|\nabla \boldsymbol{u}|}\right]\left(\boldsymbol{u}-\boldsymbol{u}^{0}\right) d x d y
$$

\section{IMPROVED MORE GENERAL TV IMAGE RESTORATION MODEL}

Using global coordinates and local coordinates of the second order directional derivative relations, a more general TV model of the diffusion equation can be expressed as:

$$
\frac{\partial \boldsymbol{u}}{\partial t}=\nabla \cdot\left(\phi^{\prime}(|\nabla \boldsymbol{u}|) \frac{\nabla \boldsymbol{u}}{|\nabla \boldsymbol{u}|}\right)-\lambda\left(\boldsymbol{u}-\boldsymbol{u}^{0}\right)=\phi^{\prime \prime}(|\nabla \boldsymbol{u}|) \boldsymbol{u}_{\eta \eta}+\frac{\phi^{\prime}(|\nabla \boldsymbol{u}|)}{|\nabla \boldsymbol{u}|} \boldsymbol{u}_{\xi \xi}(8)
$$

where, $\boldsymbol{u}_{\eta \eta}, \boldsymbol{u}_{\xi \xi}$ are the second order differential along the normal and tangential directions in the local coordinate system respectively. For digital images, the gradient value is limited, for example, a level 255 gray image of digital images, maximum and minimum gradient modulus value are

$$
|\nabla \boldsymbol{u}|_{\max }=\left(255^{2}+255^{2}\right)^{1 / 2}=255 \sqrt{2}
$$

If in plain area, the image of gradient is small. In order that the spread rates of and $|\nabla \boldsymbol{u}|_{\text {min }}=0 \quad \xi$ and $\eta$ directions are the same, it should satisfy:

$$
\phi(|\nabla \boldsymbol{u}|)=\alpha|\nabla \boldsymbol{u}|^{2}, \quad \alpha>0
$$

It can be deduced:

$$
\lim _{|\nabla \boldsymbol{u}| \rightarrow 0} \phi^{\prime \prime}(|\nabla \boldsymbol{u}|)=\lim _{|\nabla \boldsymbol{u}| \rightarrow 0} \frac{\phi^{\prime}(|\nabla \boldsymbol{u}|)}{|\nabla \boldsymbol{u}|}>0
$$

In gradient, on the other hand, the boundary area of the large gradient, we want that it spread only in the $\xi$ direction parallel to edge, and in the $\eta_{\text {direction perpendicular to edge, it stop spread or }}$ even reverse diffuse, it should satisfy at this time:

$$
\begin{gathered}
\lim _{|\nabla \boldsymbol{u}| \rightarrow 255 \sqrt{2}} \phi^{\prime \prime}(|\nabla \boldsymbol{u}|) \leq 0 \\
\lim _{|\nabla \boldsymbol{u}| \rightarrow 255 \sqrt{2}} \frac{\phi^{\prime}(|\nabla \boldsymbol{u}|)}{|\nabla \boldsymbol{u}|}>0
\end{gathered}
$$

However $\phi(|\nabla \boldsymbol{u}|)$ satisfy the type (11) at the same time is not compatible, and then we can search a suboptimal solution. Namely we hope the diffusion coefficient of $\xi$ direction and the $\eta_{\text {direction tends }}$ to zero with the increase of $|\nabla \boldsymbol{u}|$, and the diffusion rate decreases more quickly in $\eta$ direction than $\xi$ direction. So the optimal solution of the high frequency area in image is:

$$
\left\{\begin{array}{c}
\lim _{|\nabla \boldsymbol{u}| \rightarrow 255 \sqrt{2}} \phi^{\prime \prime}(|\nabla \boldsymbol{u}|) \approx 0 \\
\lim _{|\nabla \boldsymbol{u}| \rightarrow 255 \sqrt{2}} \frac{\phi^{\prime}(|\nabla \boldsymbol{u}|)}{|\nabla \boldsymbol{u}|} \approx 0 \\
\lim _{|\nabla \boldsymbol{u}| \rightarrow \infty} \frac{\phi^{\prime \prime}(|\nabla \boldsymbol{u}|)}{\phi^{\prime}(|\nabla \boldsymbol{u}|) /|\nabla \boldsymbol{u}|}<0
\end{array}\right.
$$

when

$$
\phi(|\nabla \boldsymbol{u}|)=\sqrt{1+|\nabla \boldsymbol{u}|^{2}}-1
$$

in the low frequency region $(|\nabla \boldsymbol{u}| \rightarrow 0)$, Taylor expansion of $\phi(|\nabla \boldsymbol{u}|)$ is:

$$
\phi(|\nabla \boldsymbol{u}|) \approx \frac{1}{2}|\nabla \boldsymbol{u}|^{2}
$$

It satisfies that the diffusion coefficient of $\xi$ direction and the $\eta_{\text {direction are the same in the low }}$ 
frequency region. In the high frequency region $(|\nabla \boldsymbol{u}| \rightarrow 255 \sqrt{2})$ :

$$
\left\{\begin{array}{c}
\lim _{|\nabla \boldsymbol{u}| \rightarrow 255 \sqrt{2}} \frac{\phi^{\prime}(|\nabla \boldsymbol{u}|)}{|\nabla \boldsymbol{u}|}=\frac{1}{\sqrt{1+|\nabla \boldsymbol{u}|^{2}}} \approx 0 \\
\lim _{|\nabla \boldsymbol{u}| \rightarrow 255 \sqrt{2}} \phi^{\prime \prime}(|\nabla \boldsymbol{u}|)=\frac{1}{\left(1+|\nabla \boldsymbol{u}|^{2}\right)^{3 / 2}} \approx 0 \\
\lim _{|\nabla \boldsymbol{u}| \rightarrow 255 \sqrt{2}} \frac{\phi^{\prime \prime}(|\nabla \boldsymbol{u}|)}{\phi^{\prime}(|\nabla \boldsymbol{u}|) /|\nabla \boldsymbol{u}|}=\frac{1}{1+|\nabla \boldsymbol{u}|^{2}}>0
\end{array}\right.
$$

so, it does not satisfy the third formula of optimum solution in high frequency. Namely, near the edge $(|\nabla \boldsymbol{u}| \rightarrow 255 \sqrt{2}$ ), The spread rate in the $\eta$ direction decline more slowly than the $\xi$ direction.

Therefore, we modify $\phi(\nabla \boldsymbol{u})$ functions. Make

$$
\phi(\nabla \boldsymbol{u})=\frac{\nabla \boldsymbol{u}^{2}}{1+\nabla \boldsymbol{u}^{2}}
$$

At this point, in the low frequency region $(|\nabla \boldsymbol{u}| \rightarrow 0)$, Taylor expansion of $\phi(|\nabla \boldsymbol{u}|)$ is:

$$
\phi(|\nabla \boldsymbol{u}|) \approx|\nabla \boldsymbol{u}|^{2}
$$

It satisfies that the diffusion coefficient of $\xi$ direction and the $\eta_{\text {direction are the same in the low }}$ frequency region. In the high frequency region $(|\nabla \boldsymbol{u}| \rightarrow 255 \sqrt{2})$ :

$$
\left\{\begin{array}{c}
\lim _{|\nabla \boldsymbol{u}| \rightarrow 255 \sqrt{2}} \frac{\phi^{\prime}(|\nabla \boldsymbol{u}|)}{|\nabla \boldsymbol{u}|}=\frac{2}{\left(1+|\nabla \boldsymbol{u}|^{2}\right)^{2}} \approx 0 \\
\lim _{|\nabla \boldsymbol{u}| \rightarrow 255 \sqrt{2}} \phi^{\prime \prime}(|\nabla \boldsymbol{u}|)=\frac{2\left(3|\nabla \boldsymbol{u}|^{2}-1\right)}{\left(1+|\nabla \boldsymbol{u}|^{2}\right)^{3}} \approx 0 \\
\lim _{|\nabla \boldsymbol{u}| \rightarrow 255 \sqrt{2}} \frac{\phi^{\prime \prime}(|\nabla \boldsymbol{u}|)}{\phi^{\prime}(|\nabla \boldsymbol{u}|) /|\nabla \boldsymbol{u}|}=\frac{\left(1-3|\nabla \boldsymbol{u}|^{2}\right)}{\left(1+|\nabla \boldsymbol{u}|^{2}\right)} \approx-3<0
\end{array}\right.
$$

So, when

$$
\phi(\nabla \boldsymbol{u})=\frac{\nabla \boldsymbol{u}^{2}}{1+\nabla \boldsymbol{u}^{2}}
$$

the diffusion coefficient of $\xi$ direction and the $\eta_{\text {direction tends to zero with the increase of }}|\nabla \boldsymbol{u}|$, and the diffusion rate decreases more quickly in $\eta$ direction than $\xi$ direction. In high frequency area, it still satisfies the sub-optimal solutions, in theory, it should have better diffusion performance.

\section{EXPERIMENTAL RESULTS AND ANALYSIS}

In the experiments, in order to illustrate the effectiveness of the more general TV image restoration model, we make a large number of simulation experiments, using the peak signal-tonoise ratio (PSNR) as the objective evaluation standard, selecting $256 \times 256$ blured gray image. TV model, Aubert's more general TV model as well as more general TV model of this paper has carried on for compare analysis. Table 1 shows the restoration results of Cameraman image with different intensity of gaussian noise. From table 1we can see that, the highest peak signal-to-noise are got using more general TV model of this paper. Table 2 shows the peak signal to noise ratio results of different blurred image with different models, the data in table 2 illustrates that peak signal-to-noise ratio of restored image using our model increase comparing with that using Aubert.

Table 1 Restoration results with different models using cameraman image (PSNR/dB)

\begin{tabular}{|c|c|c|c|c|}
\hline $\begin{array}{c}\text { Noise } \\
\text { intensity }\end{array}$ & $\begin{array}{c}\text { The } \\
\text { noise } \\
\text { image }\end{array}$ & $\begin{array}{c}\text { TV } \\
\text { model }\end{array}$ & $\begin{array}{c}\text { A more } \\
\text { general } \\
\text { model of } \\
\text { TV }\end{array}$ & $\begin{array}{c}\text { a more general } \\
\text { model of the } \\
\text { TV, In this } \\
\text { paper }\end{array}$ \\
\hline$\sigma^{2}=0.02$ & 22.317 & 25.632 & 26.835 & 27.128 \\
\hline$\sigma^{2}=0.04$ & 20.312 & 24.751 & 25.679 & 25.884 \\
\hline$\sigma^{2}=0.06$ & 18.872 & 24.121 & 25.578 & 25.772 \\
\hline$\sigma^{2}=0.08$ & 17.012 & 23.637 & 24.715 & 25.032 \\
\hline$\sigma^{2}=0.1$ & 16.285 & 22.291 & 23.592 & 23.723 \\
\hline
\end{tabular}

Table 2 Restoration results with different models using different images (PSNR/dB)

\begin{tabular}{|c|c|c|c|c|}
\hline $\begin{array}{c}\text { Name of } \\
\text { the image }\end{array}$ & $\begin{array}{c}\text { The } \\
\text { noise } \\
\text { image }\end{array}$ & $\begin{array}{c}\text { TV } \\
\text { model }\end{array}$ & $\begin{array}{c}\text { A more } \\
\text { general } \\
\text { model of } \\
\text { TV }\end{array}$ & $\begin{array}{c}\text { a more general } \\
\text { model of the } \\
\text { TV, In this } \\
\text { paper }\end{array}$ \\
\hline Cameraman & 20.145 & 26.721 & 27.881 & 28.218 \\
\hline Toys & 20.257 & 25.258 & 26.112 & 26.832 \\
\hline Einstein & 21.686 & 26.245 & 27.561 & 27.970 \\
\hline Lena & 20.315 & 25.248 & 26.216 & 26.632 \\
\hline Woman & 18.134 & 23.246 & 24.875 & 25.320 \\
\hline Elaine & 18.442 & 24.053 & 25.303 & 25.852 \\
\hline
\end{tabular}

To prove the subjective superiority of the more general TV model effect in this paper, select "Cameraman" and "Boat" blurred image to restore, the results is shown as in figure 1, figure 2 . It can be seen from the figure that, the TV model preserves 
the image details, but false edges exist in the smooth area; Aubert's more general TV model recovers the image effectively, but does not work well to keep edges, the improved more general TV model in this paper recovers smooth area information effectively, and reserve the high frequency part of image good, it has better visual effect.

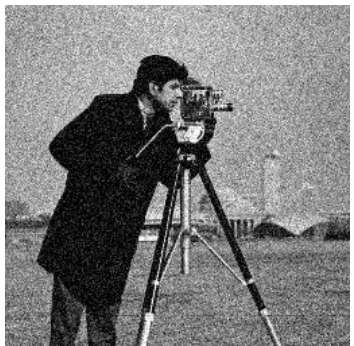

(a) The blurred image

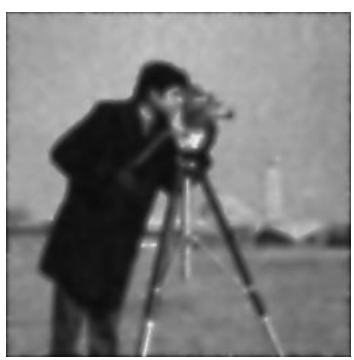

(c) Results of more general TV

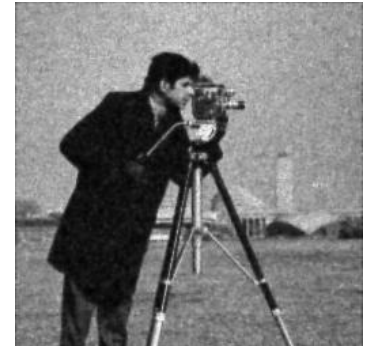

(b) The results of TV

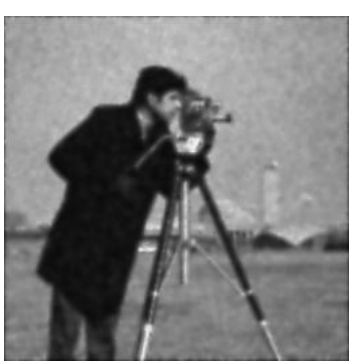

(d) Results of our TV
Figure 1. Cameraman image before and after restoration

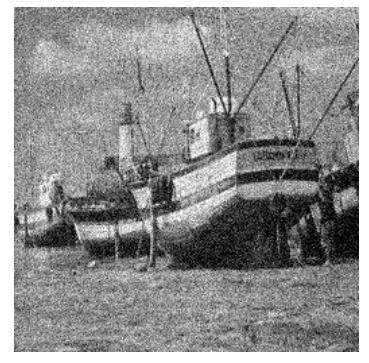

(a) The blurred image

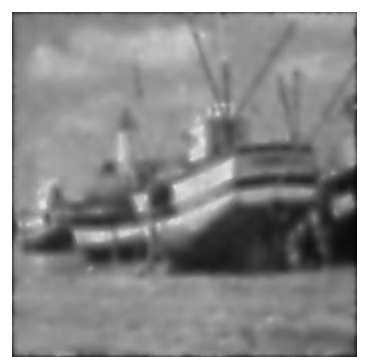

(c) Results of more general TV

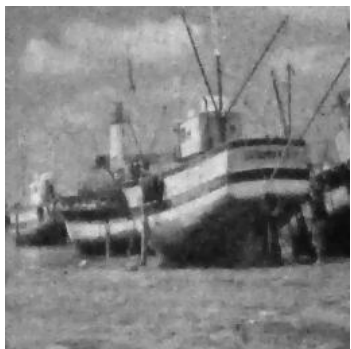

(b) The results of TV

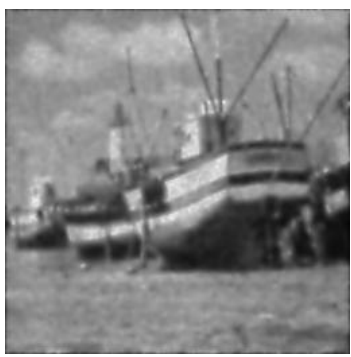

(d) Results of our TV
Figure 2. Boat image before and after restoration

\section{CONCLUSION}

In this article, through analysis of the TV image restoration model, we improve the diffusion function to get better image restoration results. Experiments results show that the new model overcome the staircase effect in smooth area and preserving significant image details in the high frequency area. This model gain higher peak signal noise ratio and has good performance of image restoration.

\section{ACKNOWLEDGMENT}

This work was supported by the Scientific Research Fund of Sichuan Provincial Artificial Intelligence Key Laboratory (2010RZ003, 2010RY003, 2011RYY02, 2011RYY04, 2012RYY05)

\section{REFERENCES}

[1] P.Perona and J.Malik. Scale-space and edge detection using anisotropic diffusion. IEEE Trans. Pat. Anal. Machine Intel.,vol. PAMI-12,no. 7, pp. 629-639, 1990.

[2] F. Catte, P. L. Lions, J. M. Morel and T. Coil. Image selective smoothing and edge detection by nonlinear diffusion, SIAM J. 1992 ,29, (1):182-193

[3] Alvarez L, Mazorra L. Signal and image restoration using shock filters and anisotropic diffusion. SIAM J Numer, Annual, 1994, 31(2): 590- 605.

[4] S.J. Osher and L. I. Rudin. Feature-Oriented Image enhancement using Shock Filters, SIAM J. Numer. Anal. 27, pp. 919-940, 1990.

[5] Chen Mingju, Yang Pingxian. With the impact a nonlinear complex diffusion filter method of image de-noising. TV technology, 2011, 35(19), 20-22

[6] L.Rudin, S.osher and E.Fatemi, Nonlinear Total Variation Based Noise Removal Algorithms, PhysieaD, 1992, 60:259-268.

[7] Chen Mingju, Yang Pingxian, Wangjing. Full fidelity term based on regularization and variational adaptive image denoising model. Journal of chongqing university of posts and telecommunications: natural science edition, 2011, 23 (5): 621-625

[8] G.Aubert and L.Vese, AVariational MethodinImage Recovery, SIAM Jounal of Numerical Analysis, 1997, 34(5): 1945-1979.

[9] Zhang Hongying, Peng Qizong. The variational adaptive image denoising model. Journal of electrical engineering, 2006, 33(3): 50-53. 